\title{
THE MOLECULAR ENVOLOPE OF MIRA
}

\author{
P. Planesas, R. Bachiller, J. Martín-Pintado, V. Bujarrabal \\ Centro Astronómico de Yebes, OAN-IGN, Spain
}

We have mapped the CO $J=2 \rightarrow 1$ and $J=1 \rightarrow 0$ emission of the circumstellar envelope of Mira. Emission in both transitions extends to a distance of $\sim 4 \times 10^{16} \mathrm{~cm}$ from the star. In the inner $2 \times 10^{16} \mathrm{~cm}$ the lines show the presence of three velocity components. The main component has the intermediate velocity and extends over the whole envelope. From this component we have estimated a mass loss rate of $3 \times 10^{-7} M_{\odot} y r^{-1}$ and a total molecular mass of $1.2 \times 10^{-3} M_{\odot}$. We have shown that, from our data, the only reliable explanation for the lowest and highest velocity components is that they are due to an outflow located within the envelope.

\section{NARROWBAND PHOTOMETRY OF PHOTOMETRICALLY PECULIAR OBJECTS}

\author{
Eugenio E. Mendosa V. \\ Instituto de Astronomia, UNAM
}

\begin{abstract}
This work is based upon $\alpha(16) \Lambda(9)$-photometry for 2 Planets, 11 Wolf-Rayet stars and 7 Planetary Nebulae. The results show anomalous $\alpha(16)$ and $\Lambda(9)$-indices for these objects. Thus, they are photometrically peculiar in this system. The main results are:

1) Callisto, Jupiter IV, shows $\alpha(16)$ and $\Lambda(9)$-indices which can be considered as excellent representatives of solar type stars (G2 V).

2) Uranus and Neptune have anomalous $\Lambda(9)$-index, because of a spectral feature in absorption around $\lambda 7805 \AA$, most likely due to a carbon compound. Thus, they are off the main sequence in the $\alpha(16) \Lambda(9)$-8rray.
\end{abstract}

3) The $\mathbf{H} \alpha$-line is possibly stronger in Uranus (marginal) and Neptune than in the Sun.

4) Wolf-Rayet stars have anomalous $\alpha(16)$-index, because of an extremely wide He II-line $(\lambda 6560 \AA)$, lack of hydrogen and the presence of spectral emission features that fall in the continuum used to determine this index.

5) Most WC stars have anomalous $\Lambda(9)$-index, because the presence of spectral emission features in the short wavelength continuum that defines this index.

6) Some W-R stars show variations in the strength of He Ir-line ( $\lambda 6560 \mathrm{~A})$.

7) Most WC stars are separated from WN stars in the $\alpha(16) \Lambda(9)$-array.

8) Planetary Nebulae have anomalous $\alpha(16)$-index, because the continuum around the hydrogen line is probably contaminated by $[\mathrm{N}$ II]-lines at $\lambda 6548 \AA$ and $\lambda 6583 \AA$.

9) Planetary Nebulae have anomalous $\Lambda(9)$-index, because the short wavelength continuum is heavily contaminated by the $[$ Ar III]-line at $\lambda 7751 \AA$.

10) Planetary Nebulae lie far apart from all kind of stellar objects in the $\alpha(16) \Lambda(9)$-diagram.

This work was partially supported by a grant from CONACYT, No. P228CCOX880202. 